\title{
Cardiorespiratory adaptations induced by aerobic training in middle-aged men: the importance of a decrease in sympathetic stimulation for the contri- bution of dynamic exercise tachycardia
}

\footnotetext{
M.P.T. Chacon-Mikahil ${ }^{1,2}$,

V.A.M. Forti ${ }^{1}$, A.M. Catai ${ }^{1,2}$,

J.S. Szrajer ${ }^{1}$, R. Golfetti ${ }^{1}$,

L.E.B. Martins ${ }^{1}$, E.C. Lima-Filho ${ }^{1}$,

J.S. Wanderley ${ }^{1}$,

J.A. Marin-Neto ${ }^{3}$, B.C. Maciel ${ }^{3}$

and L. Gallo-Jr. ${ }^{3}$
}

\author{
${ }^{1}$ Laboratório de Fisiologia do Exercício, Faculdade de Educação Física, \\ and ${ }^{2}$ Departamento de Fisiologia e Biofísica, Instituto de Biologia, \\ Universidade Estadual de Campinas, Campinas, SP, Brasil \\ ${ }^{3}$ Divisão de Cardiologia, Departamento de Clínica Médica, \\ Faculdade de Medicina de Ribeirão Preto, Hospital das Clínicas, \\ Universidade de São Paulo, Ribeirão Preto, SP, Brasil
}

Correspondence

L. Gallo-Jr.

Divisão de Cardiologia

Departamento de Clínica Médica Hospital das Clínicas, FMRP, USP 14049-900 Ribeirão Preto, SP Brasil

Fax: 55 (016) 633-1144

Research supported by FAPESP (Nos. 89/1297-6, 91/4754-9) and FAEP-UNICAMP (No. 1055/91).

Received April 9, 1997 Accepted January 15, 1998

\section{Abstract}

We investigated the effects of aerobic training on the efferent autonomic control of heart rate (HR) during dynamic exercise in middleaged men, eight of whom underwent exercise training $(\mathrm{T})$ while the other seven continued their sedentary (S) life style. The training was conducted over 10 months (three 1-h/sessions/week on a field track at $70-85 \%$ of the peak HR). The contribution of sympathetic and parasympathetic exercise tachycardia was determined in terms of differences in the time constant effects on the HR response obtained using a discontinuous protocol (4-min tests at 25, 50, 100 and 125 watts on a cycle ergometer), and a continuous protocol ( 25 watts/min until exhaustion) allowed the quantification of the parameters (anaerobic threshold, $\dot{\mathrm{V}} \mathrm{O}_{2} \mathrm{AT}$; peak $\mathrm{O}_{2}$ uptake, $\dot{\mathrm{VO}}_{2}$ peak; power peak) that reflect oxygen transport. The results obtained for the $\mathrm{S}$ and the $\mathrm{T}$ groups were: 1) a smaller resting HR in T (66 beats/min) when compared to $S$ ( 84 beats/min); 2) during exercise, a small increase in the fast tachycardia $(\Delta 0-10 \mathrm{~s})$ related to vagal withdrawal ( $\mathrm{P}<0.05$, only at 25 watts) was observed in $\mathrm{T}$ at all powers; at middle and higher powers a significant decrease $(\mathrm{P}<0.05$ at 50,100 and 125 watts $)$ in the slow tachycardia ( $\Delta 1-4 \mathrm{~min})$ related to a sympathetic-dependent mechanism was observed in $\mathrm{T} ; 3)$ the $\dot{\mathrm{VO}} \mathrm{O}_{2} \mathrm{AT}(\mathrm{S}=1.06$ and $\mathrm{T}=1.33 \mathrm{l} / \mathrm{min})$ and $\dot{\mathrm{V}} \mathrm{O}_{2}$ peak $(\mathrm{S}=1.97$ and $\mathrm{T}=2.47 \mathrm{l} / \mathrm{min})$ were higher in $\mathrm{T}(\mathrm{P}<0.05)$. These results demonstrate that aerobic training can induce significant physiological adaptations in middle-aged men, mainly expressed as a decrease in the sympathetic effects on heart rate associated with an increase in oxygen transport during dynamic exercise.
Key words

- Sympathetic modulation of heart rate

- Dynamic exercise

- Aerobic training

- Autonomic nervous system

- Anaerobic threshold

- Middle-aged men 


\section{Introduction}

The cardiorespiratory system at rest and during exercise is usually studied by monitoring variables such as heart rate (HR) and oxygen transport $\left(\mathrm{VO}_{2}\right)$. HR in particular is considered to be an important variable in the quantification of certain physiological properties of the cardiovascular system. This variable has the advantage that it can be determined readily with minimum error using noninvasive methods $(1,2)$.

The ability to continuously monitor HR permits the use of dynamic exercise in functional evaluations of the two components of the cardiac autonomic nervous system, as suggested by the investigations conduced with or without pharmacological blockade on sedentary or trained men (3-6). Such studies suggest that tachycardia evoked by dynamic exercise is mediated by a biphasic mechanism which initially involves vagal withdrawal (very fast increase of HR), and increased delayed sympathetic activity participates as an additional component at higher powers (slow linear HR increase) $(1,3,4)$.

Oxygen transport is a complex process that involves the participation of several physiological components such as the cardiorespiratory system, transport mechanisms (hemoglobin and myoglobin) and oxidative enzymes. The two parameters that best reflect the functioning of this process are maximal oxygen consumption $\left(\mathrm{VO}_{2}\right.$ max $)$ and ventilatory anaerobic threshold (AT). Since the direct measurement of $\mathrm{VO}_{2}$ max is very difficult to obtain under normal and pathological conditions, the AT provides a useful means of measuring oxygen transport mainly because it can be easily determined at submaximal powers by noninvasive methods. In addition, the AT allows a suitable separation of two important physiological states: 1) below the AT, the cardiorespiratory responses are linear and most variables reach a steadystate condition, and 2) above the AT, the respective responses assume a nonlinear be- havior in which the variables do not fully stabilize $(1,7,8)$.

Several studies have shown that maximal aerobic capacity reaches a maximum around the age of 30 years and that it decreases progressively thereafter. Limitations related to a reduction in maximum cardiac output appear to play an important role in age-dependent changes in aerobic transport during exercise (9-13). While many studies have characterized the cardiovascular responses in middleaged men, the autonomic control of HR in this particular group during exercise has received much less attention (12-18).

In the present study we determined whether aerobic training induced significant adaptations in the sympathetic and parasympathetic control of HR during dynamic exercise in middle-aged men.

\section{Material and Methods}

Subjects

Fifteen men (46-60 years old; mean \pm $\mathrm{SD}, 50.7 \pm 3.6)$ were studied. The characteristics of the groups are shown in Table 1. All volunteers were in good health, and had no evidence of cardiovascular disease on the basis of clinical and laboratory evaluation that included the analysis of standard electrocardiograms and chest X-rays. The subjects were not taking any medication and led a relatively sedentary life style, although some of them occasionally participated in week-end sports. During the study, seven subjects continued to lead a sedentary life while the remaining eight underwent an aerobic training program. All volunteers gave informed consent to participate in this investigation. The study was approved by the Ethics Committee of the University Hospital of the State University of Campinas.

Aerobic training

Eight sedentary subjects underwent a 10- 
month aerobic training program consisting of walking and jogging on a field track three times a week for $1 \mathrm{~h}$. The intensity of exercise was set at $70-85 \%$ of the peak heart rate (HRpeak) obtained in a previous exercise session (sitting on a cycle ergometer) performed in the laboratory.

Experimental protocols

The subjects were studied at rest (supine position) and during dynamic exercise tests on a cycle ergometer. In all the protocols, the electrocardiogram (ECG) was continuously monitored using the precordial MC-5 lead. Before the tests, the subjects were allowed to familiarize themselves with the procedures and the equipment to be used. The sessions were held 2-3 $\mathrm{h}$ after a light meal at an environmental temperature of around $23^{\circ} \mathrm{C}$.

The resting HR was obtained after 20 min of resting in the supine position. The HR values obtained here and immediately before (1 $\mathrm{min}$ ) and during exercise testing (in a sitting position) are reported as the mean determined at 10-s intervals by counting the number of QRS complexes directly from the ECG tracing using a calibrated ruler.

The dynamic exercise was always performed in the sitting position using an electromagnetic braked cycle ergometer model Ciclo II (FUNBEC, São Paulo, SP, Brazil). Two exercise protocols were employed. In the discontinuous protocol workloads corresponding to $25,50,100$ and 125 watts ( $4 \mathrm{~min}$ each) were used with a frequency of $60 \mathrm{rpm}$ being maintained throughout each stage. Varying resting periods were allowed between the different powers so that HR could return to control (basal) levels. The electrocardiogram was recorded continuously from $30 \mathrm{~s}$ before the beginning to $1 \mathrm{~min}$ after the end of effort using a one-channel recording unit model ECG40A (FUNBEC, Brazil).

The main objective of this protocol was to evaluate the contribution of the sympathetic and parasympathetic systems to the
Table 1 - Anthropometric characteristics of the groups studied.

\begin{tabular}{llccc}
\hline $\begin{array}{l}\text { Physical } \\
\text { condition }\end{array}$ & & $\begin{array}{c}\text { Weight } \\
(\mathrm{kg})\end{array}$ & $\begin{array}{c}\text { Height } \\
(\mathrm{cm})\end{array}$ & $\begin{array}{c}\text { Body surface } \\
\text { area }\left(\mathrm{m}^{2}\right)\end{array}$ \\
\hline $\begin{array}{l}\text { Sedentary } \\
(\mathrm{N}=7)\end{array}$ & minimum & 61 & 163 & 1.64 \\
& 1st quartile & 64 & 168 & 1.75 \\
& median & 76 & 172 & 1.88 \\
& 3rd quartile & 81 & 175 & 1.95 \\
& maximum & 85 & 184 & 2.08 \\
Trained & minimum & 72 & 167 & 1.84 \\
$(\mathrm{~N}=8)$ & 1st quartile & 78 & 170 & 1.89 \\
& median & 81 & 174 & 1.95 \\
& 3rd quartile & 83 & 176 & 1.98 \\
& maximum & 93 & 180 & 2.10
\end{tabular}

increase in HR induced by dynamic exercise and was based on the time constant differences of the two divisions of the autonomic nervous system when modulating HR changes during dynamic exercise. This method was validated in previous studies in which the HR response induced by exercise was evaluated before and after selective pharmacological blockade of the sympathetic and parasympathetic systems $(1,3,4)$.

The continuous protocol was performed by progressively increasing power steps until physical exhaustion. The objective of this protocol was to evaluate aerobic capacity by measuring the $\operatorname{AT}(7,19)$ at submaximal work loads and the $\mathrm{VO}_{2}$ peak at physical exhaustion. After a 2-min warm-up period (at 5 watts), the power was increased by 25 watts each minute (steps) until the volunteers became physically exhausted. The researcher closely monitored the signals from the subject (facial expression, blood pressure and abnormal ECG recordings) and always verbally encouraged the volunteer to maximize his performance. The test exercise ended when the subject was unable to maintain the standard cycling frequency. To avoid syncope after reaching peak power, the subjects continued the exercise at a lower frequency and power for approximately $2 \mathrm{~min}$.

During both experimental protocols, the subjects breathed through a low-resistance, non-rebreathing, two-way valve model 2700 
Table 2 - Functional data of the subject groups studied.

\begin{tabular}{llccc}
\hline $\begin{array}{l}\text { Physical } \\
\text { condition }\end{array}$ & & $\begin{array}{c}\text { HR resting } \\
\text { (beats/min) }\end{array}$ & $\begin{array}{c}\text { HR peak } \\
\text { (beats/min) }\end{array}$ & $\begin{array}{c}\text { Peak power } \\
\text { (watts) }\end{array}$ \\
\hline $\begin{array}{l}\text { Sedentary } \\
(\mathrm{N}=7)\end{array}$ & minimum & 60 & 144 & 100 \\
& 1st quartile & 60 & 150 & 137 \\
& median & 84 & 156 & 150 \\
& 3rd quartile & 90 & 165 & 187 \\
& maximum & 96 & 180 & 200 \\
Trained & minimum & 54 & 144 & 175 \\
$(\mathrm{~N}=8)$ & 1st quartile & 60 & 166 & 219 \\
& median & 66 & 176 & 237 \\
& 3rd quartile & 72 & 181 & 250 \\
& maximum & 84 & 192 & 275
\end{tabular}

(Hans Rudolph, Kansas City, MO), and the expired gas was analyzed continuously using a metabolic measuring system (MMC Horizon Systems, Sensor Medics, Yorba Linda, CA). Average values for ventilation $(\dot{V})$, oxygen uptake $\left(\dot{\mathrm{VO}}_{2}\right)$, carbon dioxide output $\left(\mathrm{VCO}_{2}\right)$, and respiratory exchange ratio (RER) were calculated automatically at 15 -s intervals.

The peak of each variable was selected as the highest value reached during the incremental exercise period since, as a result of muscle fatigue, the true $\mathrm{VO}_{2}$ max was never attained. This was indicated by the lack of a plateau (saturation) in oxygen uptake during exercise (7).

Using the ventilatory and gas exchange responses during the last exercise test it was possible to obtain a measurement of AT for all subjects with this noninvasive method. These values, reported as $\dot{\mathrm{VO}}_{2}(1 / \mathrm{min})$, were determined using the criterion of a nonlinear increase in $\mathrm{V}$ and $\mathrm{VCO}_{2}$ in comparison to the linear increase in $\mathrm{VO}_{2}$ at submaximal work loads $(7,19,20)$. The AT values related to $\dot{\mathrm{VO}}_{2}$ peak $\left(\mathrm{AT} \% \dot{\mathrm{VO}}_{2}\right.$ peak) were also calculated.

Statistical analysis

Data are reported as medians, quartiles (1st and 3rd) and minimum and maximum values. The Mann-Whitney nonparametric test was used to compare the variables and parameters between the two studied groups. The level of significance was set at $5 \%$.

\section{Results}

The resting HR and the HR and power peaks during exercise for the sedentary and trained groups are shown in Table 2. The trained group had lower median values than the sedentary group under resting conditions but the difference was not statistically significant. An inverse non-significant situation was also observed for HRpeak. Under both conditions, the difference between the two groups was approximately 20 beats $/ \mathrm{min}$.

The increases in HR $(\Delta H R)$ during dynamic exercise in the sedentary and trained groups are presented in Figures 1, 2 and 3.

In spite of the higher median values observed in the trained group during the 0-10$\mathrm{s}$ interval $(\Delta 0-10 \mathrm{~s})$, the difference was only significant at a power value of 25 watts $(\mathrm{P}=$ 0.05) (Figure 1). On the other hand, the 1-4 min median increases in HR (Figure 2) were, with one exception ( 25 watts, $\mathrm{P}=0.24$ ), lower in the trained than in the sedentary group at all power values: 50 watts $(\mathrm{P}=$ $0.04), 100$ watts $(\mathrm{P}=0.04)$ and 125 watts $(\mathrm{P}$ $=0.01)$. For the $0-4$ min increases in $\mathrm{HR}(\Delta 0$ $4 \mathrm{~min}$ ), the median values of the two groups were similar at all powers studied (Figure 3 ).

Figure 4 illustrates several parameters that reflect the magnitude of oxygen transport at submaximal $\left(\dot{\mathrm{VO}}_{2} \mathrm{AT}\right)$ and peak $\left(\dot{\mathrm{VO}}_{2}\right.$ peak) powers. The values for $\mathrm{V}_{2} \mathrm{AT}$ (sedentary, $1.06 \mathrm{l} / \mathrm{min}$; trained, $1.33 \mathrm{l} / \mathrm{min} ; \mathrm{P}=$ 0.02) and $\dot{\mathrm{VO}}_{2}$ peak (sedentary, $1.97 \mathrm{l} / \mathrm{min}$; trained, $2.47 \mathrm{l} / \mathrm{min}$; $\mathrm{P}=0.01$ ) were significantly higher in the trained group. However, when the AT was expressed as a percentage of $\dot{\mathrm{V}}_{2}$ peak $\left(\mathrm{AT} \% \dot{\mathrm{V}}_{2}\right.$ peak) the results were similar for both groups.

\section{Discussion}

The resting HR is modulated by a bal- 
ance between sympathetic and parasympathetic tone, with the latter predominating. Although the resting HR does not change with increasing age, Lakatta (21) has reported a decrease in the respiratory variation of HR, probably caused by a reduction in parasympathetic and sympathetic modulation. In the presence of both sympathetic and parasympathetic blockade, the intrinsic sinus node rate decreases significantly with age: at the age of 20 years, the average intrinsic HR is 104 beats/min compared with 92 beats/min in the $45-55$-year-old group (22). In the middle-aged men studied here, aerobic training was expected to cause a greater reduction in resting $\mathrm{HR}$ than that actually observed; the absence of a statistically significant difference in our data probably reflects the small number of subjects studied. Regardless of these considerations, the magnitude of bradycardia found in middle-aged men was comparable to that reported by others $(14,15)$. It should be emphasized that this adaptation in HR is usually considered to be a good marker of adequate training (10,23-26) and, at least in men, an intrinsic mechanism seems to play a predominant role in the respective adaptation (27-29).

Since in the resting state the cardiovascular system usually operates below its full functional reserve, studies performed in this situation cannot adequately characterize the full extent of the regulatory mechanisms involved.

In the present investigation, we studied the HR responses during exercise and their relation to the autonomic control of HR using a nonpharmacological method based on the time constant differences for the activation of each efferent limb of the autonomic nerves acting on the sinus node $(3,4,30,31)$. The fast tachycardia seen after the start of exercise occurs during the initial $10 \mathrm{~s}$ of activity at all levels of exercise and is mediated by a sudden reduction in vagal tone on the sinus node $(3,31,32)$. The HR increase in

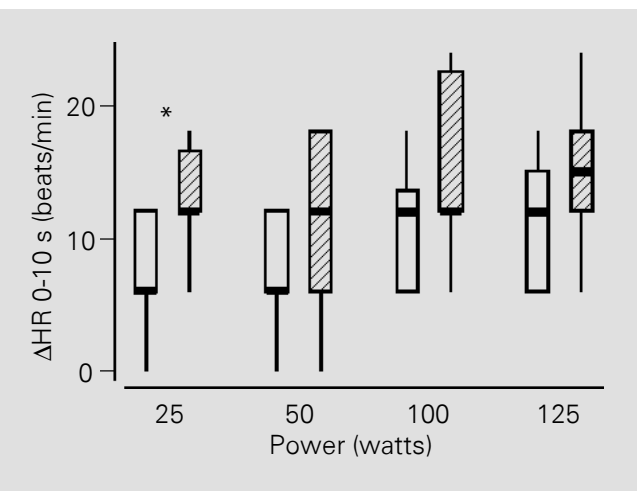

Figure 1 - Increases in $\mathrm{HR}(\Delta \mathrm{HR})$ during dynamic exercise at power values of $25,50,100$ and 125 watts, in the $0-10$-s interval $(\Delta 0-10 \mathrm{~s})$ in the sedentary (open boxes, $\mathrm{N}=7$ ) and trained (hatched boxes, $\mathrm{N}=8$ ) groups. The data are reported as medians, quartiles (1st and 3rd) and minimum and maximum values. ${ }^{*} \mathrm{P}<0.05$.

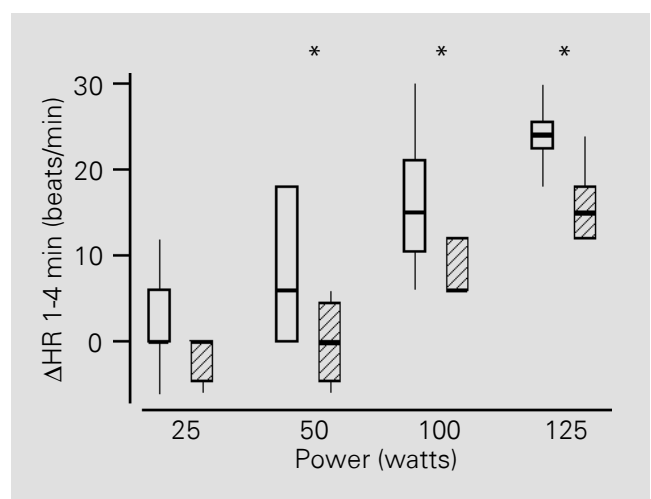

Figure 2 - Increases in $\mathrm{HR}(\Delta \mathrm{HR})$ during dynamic exercise at power values of $25,50,100$ and 125 watts, in the 1-4-min interval ( $\Delta 1-4 \mathrm{~min})$ in the sedentary (open boxes, $\mathrm{N}=7$ ) and trained (hatched boxes, $\mathrm{N}=8$ ) groups. The data are reported as medians, quartiles (1st and 3rd) and minimum and maximum values. ${ }^{*} \mathrm{P}<0.05$.

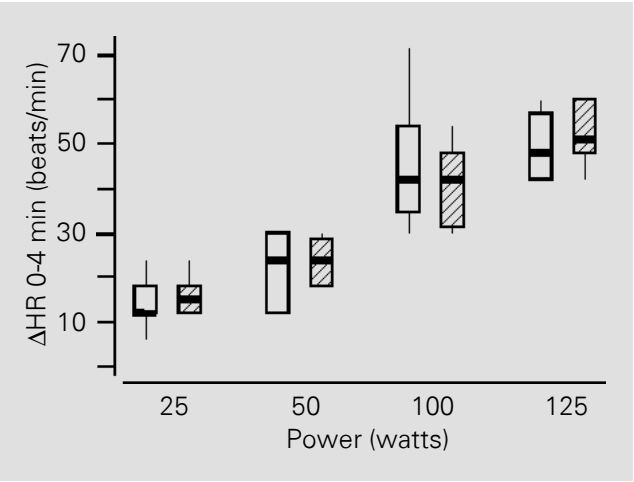

Figure 3 - Increases in $\mathrm{HR}(\Delta \mathrm{HR})$ during dynamic exercise at power values of 25,50, 100 and 125 watts, in the $0-4$-min interval $(\Delta 0-4 \mathrm{~min})$ in the sedentary (open boxes, $\mathrm{N}=7$ ) and trained (hatched boxes, $\mathrm{N}=8$ ) groups. The data are reported as medians, quartiles (1st and 3rd) and minimum and maximum values.

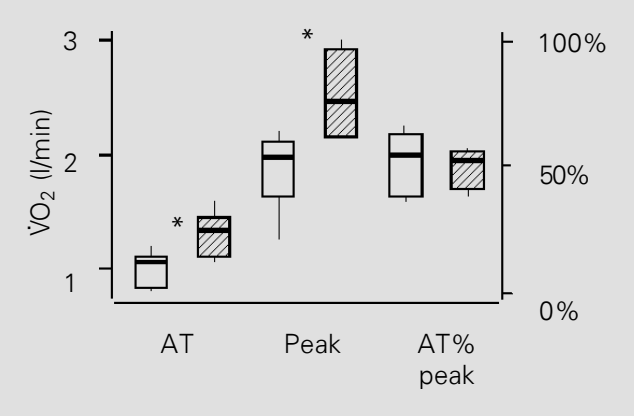

Figure 4 - The anaerobic threshold $\left(\mathrm{VO}_{2} \mathrm{AT}\right)$, peak oxygen uptake $\left(\mathrm{VO}_{2}\right.$ peak) and the AT related to $\quad \dot{\mathrm{VO}} 2$ peak (AT\% $\quad \dot{\mathrm{VO}}_{2}$ peak) values during a continuous protocol in the sedentary (open boxes, $\mathrm{N}=7$ ) and trained (hatched boxes, $\mathrm{N}=8$ ) groups. The data are reported as medians, quartiles (1st and 3rd) and minimum and maximum values. ${ }^{*} \mathrm{P}<0.05$. 
the $\Delta 0$-10-s interval (vagal-dependent) found in our study differed little between groups, indicating simply a minor adaptation of vagal mechanisms after training.

On the other hand, the slow increase in HR ( $\Delta$ 1-4 min) during exercise was significantly lower in the trained group. This finding indicates that aerobic training can induce a substantial reduction in sympathetic stimulation during dynamic exercise at power levels above the anaerobic threshold $(1,4)$. The nonsignificant difference in the $\Delta 1-4 \mathrm{~min}$ increases in HR at 25 watts in both groups is explained by the fact that at this low intensity workload the exercise tachycardia predominantly reflects parasympathetic withdrawal on the sinus node (1).

The absence of any change in total HR, i.e., from 0 to $4 \mathrm{~min}$, reinforces the importance of measuring the fractional contribution of exercise tachycardia caused by each efferent division of the autonomic nervous system. Overall, our results for this age group are similar to those obtained for younger subjects $(3,31)$.

In agreement with others $(14,15)$, we observed small nonsignificant differences in HRpeak between groups. The values in both groups were lower than those found in younger subjects $(9,33-36)$. This decrease in the HRpeak during exercise in older individuals is usually independent of the degree of aerobic performance or of a concomitant disease, since a deficit of similar magnitude is seen in both healthy sedentary men and women as well as in older athletes $(16,34,37$, 38).

Numerous studies have described the changes in cardiovascular function occurring with age, particularly during acute exercise $(9,13,15,21,36,39-41)$. Older individuals show a lower aerobic capacity, and have several other noncardiovascular factors that can limit their physical performance, including orthopedic difficulties, alterations in body composition, a reduction in muscle mass and strength, a lower threshold for neuromuscu- lar fatigue and so on (21).

It is well known that $\mathrm{VO}_{2}$ max and $\mathrm{VO}_{2}$ peak decline with age as reported in longitudinal and cross-sectional studies (9,39-41). The maximum cardiac output also declines progressively because of an associated reduction in maximum HR $(17,21)$. An important point to be considered is that the $\dot{\mathrm{VO}}_{2}$ max or $\dot{\mathrm{V}}_{2}$ peak can be improved with exercise training of sufficient duration, frequency, and intensity $(15,17,18,34,42,43)$. The increase in $\mathrm{VO}_{2}$ peak in our trained group was comparable to that reported by others and can reflect an important ability for adaptation at all ages $(14,15,17)$.

The major concern in exercise testing should be to determine whether the cardiorespiratory system is capable of providing the required amount of oxygen to the exercise-stressed muscles (7). However, for methodological reasons in many situations it is impossible to obtain the values of $\mathrm{VO}_{2}$ max that are needed to characterize oxygen transport during maximal exercise. In such circumstances, the anaerobic threshold is the best alternative to solve this problem.

The AT is defined as the power level of exercise above which aerobic energy production is supplemented by anaerobic mechanisms. In this condition, there is an increase in lactate and in the lactate/pyruvate ratio in muscle and arterial blood. AT shows a good correlation with $\mathrm{VO}_{2}$ max and has proved to be very useful in quantifying oxygen transport and its modification by physiological and pathological conditions (7).

Particularly important are the changes in AT values induced by aging and aerobic training. The absolute median AT values of our trained subjects were higher than those of the sedentary group, as also reported elsewhere $(44,45)$. However, when AT was expressed as a percentage of the $\mathrm{VO}_{2}$ peak, there was no difference between groups. The reason for this finding is unclear.

The responses of cardiorespiratory variables to a step exercise test require 2-3 min 
to reach a steady state. Indeed, the full stabilization of cardiorespiratory variables is never reached during exercise above the AT (1). This nonstationary response of $\mathrm{HR}$ is related to the occurrence of sympathetic drive. The progressive increase in $\Delta 1-4 \mathrm{~min} H R$, with increasing power values reflects the proportional elevation of sympathetic stimulation at high workloads. In this context, our findings have shown a displacement of the AT toward higher workloads in middle-aged men similar to that occurring with the beginning and the intensification of sympathetic stimulation of the sinus node during exercise.

The present results have shown that aerobic training induces significant physiological adaptations in the cardiorespiratory system of middle-aged men. The best markers of these adaptations were the smaller sympa- thetic tachycardia ( $\Delta 1-4 \mathrm{~min})$ at comparable workloads and the improvement of oxygen transport, as documented by the increase in the anaerobic threshold and $\mathrm{VO}_{2}$ peak during dynamic exercise.

\section{Acknowledgments}

The authors are grateful to the Departamento de Clínica Médica and the Seção de Cardiologia, Faculdade de Ciências Médicas, Universidade Estadual de Campinas (UNICAMP), where the clinical examination and standard ergometric and biochemical tests were performed. We are also indebted to Cleide Marques Antloga and Pedro Mikahil Neto for a final revision of the manuscript.

\section{References}

1. Gallo-Jr L, Maciel BC, Marin-Neto JA, Martins LEB, Lima-Filho EC, Golfetti R, Chacon MPT \& Forti VAM (1995). Control of heart rate during exercise in health and disease. Brazilian Journal of Medical and Biological Research, 28: 1179-1184.

2. Miyamoto $Y$, Hiura T, Tamura T, Nakamura T, Higuchi J \& Mikami T (1982). Dynamics of cardiac, respiratory and metabolic function in men in response to step workload. Journal of Applied Physiology, 52: 11981208.

3. Maciel BC, Gallo-Jr L, Marin-Neto JA, Lima-Filho EC \& Martins LEB (1986). Autonomic nervous control of the heart rate during dynamic exercise in normal man. Clinical Science, 71: 457-460

4. Gallo-Jr L, Maciel BC, Marin-Neto JA \& Martins LEB (1989). Sympathetic and parasympathetic changes in heart rate control during dynamic exercise induced by endurance training in man. Brazilian Journal of Medical and Biological Research, 22: 631-643.

5. Chacon MPT, Forti VAM, Catai AM, Szrajer JS, Paschoal MA, Golfetti R, Martins LEB, Maciel BC, Marin-Neto JA, LimaFilho EC, Wanderley JS \& Gallo-Jr L (1994). Cardiorespiratory adaptation to aerobic training in middle-aged men. Physics in Medicine and Biology, 39a (Part 1): 115 (Abstract)
6. Forti VAM, Chacon MPT, Catai AM, Szrajer JS, Paschoal MA, Golfetti R, Martins LEB, Maciel BC, Marin-Neto JA, LimaFilho EC, Wanderley JS, Sztejnsznajd CA \& Gallo-Jr L (1994). The effects of aerobic training on cardiovascular system in menopause. Physics in Medicine and Biology, 39a (Part 1): 113 (Abstract).

7. Wasserman K, Hansen JE, Sue DY, Whipp BJ \& Casaburi R (1994). Principles of Exercise Testing and Interpretation. 2nd edn. Lea \& Febiger, Philadelphia.

8. Brooks GA (1991). Current concepts in lactate exchange. Medicine and Science in Sports and Exercise, 23: 895-906.

9. Robinson S (1938). Experimental studies of physical fitness in relation to age. Arbeitsphysiologie, 10: 251-323.

10. Saltin B \& Rowell LB (1980). Functional adaptations to physical activity and inactivity. Federation Proceedings, 39: 15061513.

11. Sutton JR (1992). $\dot{V} \mathrm{O}_{2}$ max. New concepts on an old theme. Medicine and Science in Sports and Exercise, 24: 26-29.

12. Inbar $O$, Oren $A$, Scheinowitz $M$, Rotstein A, Dlin R \& Casaburi R (1994). Normal cardiopulmonary responses during incremental exercise in 20- to 70-year-old men. Medicine and Science in Sports and Exercise, 26: 538-546.

13. Jackson A, Beard EF, Wier LT, Ross RM,
Stuteville JE \& Blair SN (1995). Changes in aerobic power of men, ages 25-70yr. Medicine and Science in Sports and Exercise, 27: 113-120.

14. Hanson JS, Tabakin BS, Levy AM \& Nedde W (1968). Long-term physical training and cardiovascular dynamics in middle-aged men. Circulation, 38: 783799.

15. Hagberg JM (1987). Effect of training on the decline of $\mathrm{VO}_{2}$ max with aging. Federation Proceedings, 46: 1830-1833.

16. Heath GW, Hagberg JM, Ehsani AA \& Holloszy JO (1981). A physiological comparison of young and older endurance athletes. Journal of Applied Physiology, 51: 634-640.

17. Posner JD, Gorman KM \& Klein HS (1986) Exercise capacity in the elderly. American Journal of Cardiology, 57: 52C-58C.

18. Ehsani AA (1987). Cardiovascular adaptations to exercise training in the elderly. Federation Proceedings, 46: 1840-1843.

19. Beaver WL, Wasserman K \& Whipp BJ (1986). A new method for detecting anaerobic threshold by gas exchange. Journal of Applied Physiology, 60: 20202027

20. Wasserman K, Beaver WL \& Whipp BJ (1990). Gas exchange theory and the lactic acidosis (anaerobic) threshold. Circulation, 81 (Suppl II): II-14-II-30. 
21. Lakatta EG (1993). Cardiovascular regulatory mechanisms in advanced age. Physiological Reviews, 73: 413-467.

22. Jose AD (1966). Effect of combined sympathetic and parasympathetic blockade on heart rate and cardiac function in man. American Journal of Cardiology, 18: 476478.

23. Negrão CE, Forjaz CLM, Rondon MUPB \& Brum PC (1996). Adaptação cardiovascular ao treinamento físico dinâmico. In: Souza AGM \& Mansur AJ (Editors), Socesp Cardiologia. Vol. 2. Atheneu, São Paulo.

24 Åstrand P-O \& Rodahl K (1980). Tratado de Fisiologia do Exercício. 2nd edn. Interamericana, Rio de Janeiro.

25. Ekblom B, Kilbom A \& Stoltysiak J (1973). Physical training, bradycardia and autonomic nervous system. Scandinavian Journal of Clinical and Laboratory Investigation, 32: 251-256.

26. Lewis $\mathrm{SF}$, Nylander E, Gad P \& Areskog $\mathrm{NH}$ (1980). Non autonomic component in bradycardia of endurance trained men at rest and during exercise. Acta Physiologica Scandinavica, 109: 297-305.

27. Katona PG, McLean M, Dighton DH, Davis HD \& Guz A (1982). Sympathetic and parasympathetic cardiac control in athletes and non-athletes at rest. Journal of Applied Physiology: Respiratory, Environmental and Exercise Physiology, 52: 16521657.

28. Maciel BC, Gallo-Jr L, Marin-Neto JA, Lima-Filho EC, Terra-Filho J \& Manço JC (1985). Parasympathetic contribution to bradycardia induced by endurance training in man. Cardiovascular Research, 19: 642-648.

29. Perrault H, Gagnon MC, Johnson D,
Mokrane A \& Nadeau RA (1996). An enhanced vagal influence does not explain training induced bradycardia. Physiologist, 39: 20 (Abstract).

30. Maciel BC, Gallo-Jr L, Marin-Neto JA, Maciel LMZ \& Martins LEB (1988). Autonomic control of heart rate during dynamic exercise in human hyperthyroidism. Clinical Science, 75: 209-215.

31. Gallo-Jr L, Morelo-Filho J, Maciel BC, Marin-Neto JA, Martins LEB \& Lima-Filho EC (1987). Functional evaluation of sympathetic and parasympathetic system in Chagas' disease using dynamic exercise. Cardiovascular Research, 21: 922-927.

32. Fagraeus L \& Linnarsson D (1976). Autonomic origin of heart rate fluctuations at the onset of muscular exercise. Journal of Applied Physiology, 40: 679-682.

33. Landeree BR, Thomas TR, Ziogas G, Smith TD \& Zhang O (1995). \% $\dot{V} \mathrm{O}_{2}$ max versus \% HRmax regressions for six modes of exercise. Medicine and Science in Sports and Exercise, 27: 458-461.

34. Åstrand I (1960). Aerobic work capacity in men and women with special reference to age. Acta Physiologica Scandinavica, 49 (Suppl 169): 1-92.

35. Hammond HK \& Froelicher VF (1985). Normal and abnormal heart rate responses to exercise. Progress in Cardiovascular Diseases, 27: 271-296.

36. Babcock MA, Paterson DH \& Cunningham DA (1994). Effects of aerobic endurance training on gas exchange kinetics of older men. Medicine and Science in Sports and Exercise, 26: 447-452.

37. Fleg JL, Schulman S, Gerstenblith G, Goldber A, Tankersley C, Becker L, Clulow J, Drinkwater D, Lakatta L \& Lakatta EG (1988). Central versus peripheral adapta- tions in highly trained seniors. Physiologist, 31: 158 (Abstract).

38. Ehsani AA, Ogawa T, Miller TR, Spina RJ \& Jilka SM (1991). Exercise training improves left ventricular systolic function in older men. Circulation, 83: 96-103.

39. Lakatta EG (1995). Cardiovascular system. In: Masoro EJ (Editor), Handbook of Physiology. A Critical, Comprehensive Presentation of Physiological Knowledge and Concepts. Section 11: Aging. Oxford University Press, New York.

40. Åstrand I, Åstrand P-O, Hallback I \& Kilbom $\AA$ (1973). Reduction in maximal oxygen uptake with age. Journal of Applied Physiology, 35: 649-654.

41. Buskirk ER \& Hodgson JL (1987). Age and aerobic power: the rate of change in men and women. Federation Proceedings, 46: 1824-1829.

42. Pollock ML, Foster C, Knapp D, Rod JL \& Schmidt DH (1987). Effect of age and training on aerobic capacity and body composition of master athletes. Journal of Applied Physiology, 62: 725-731.

43. Kasch FW, Boyer JL, VanCamp SP, Verity LS \& Wallace J (1990). The effect of physical activity and inactivity on aerobic power in older men (a longitudinal study). Physician Sportsmedicine, 18: 73-81.

44. Davis JA, Frank MH, Whipp BJ \& Wasserman K (1979). Anaerobic threshold alteration caused by endurance training in middle-aged men. Journal of Applied Physiology, 46: 1039-1046.

45. Thomas SG, Cunningham DA, Thompson NJ \& Rechnitzer PA (1985). Exercise training and "ventilation threshold" in elderly. Journal of Applied Physiology, 59: 14721476. 